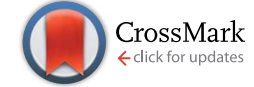

Cite this: RSC Adv., 2017, 7, 11568

Received 1st January 2017

Accepted 1st February 2017

DOI: $10.1039 / \mathrm{c} 7 \mathrm{ra00007c}$

rsc.li/rsc-advances

\section{A free standing porous Co/Mo architecture as a robust bifunctional catalyst toward water splitting $\dagger$}

\author{
Yuxuan Li, ${ }^{\mathrm{b}}$ Xingbo Ge, ${ }^{\text {ab }}$ Leidanyang Wang, ${ }^{\mathrm{b}}$ Jia Liu, ${ }^{\mathrm{b}}$ Yong Wang ${ }^{\mathrm{b}}$ \\ and Lanxiang Feng ${ }^{b}$
}

A macroporous Co/Mo-based composite was fabricated for the first time by etching its alloy in ceric ammonium nitrate. It was used directly as a free standing electrode and exhibited remarkable catalytic activities for both the oxygen evolution reaction and the hydrogen evolution reaction, making it a robust bifunctional catalyst for water splitting.
Exploring renewable and clean energy has always been one of the hottest issues for researchers worldwide. As a type of sustainable and highly efficient energy, hydrogen $\left(\mathrm{H}_{2}\right)$ has raised tremendous expectations. Hydrogen production from water splitting is regarded as an innovative approach to store energy obtained from other intermittent sources such as the Sun. ${ }^{1}$ A suitable catalyst is required to largely decrease the overpotentials that arise from the uphill reaction nature. Currently, Pt-based composites are the most active catalysts for the hydrogen evolution reaction (HER) at the cathode. $\mathrm{RuO}_{2}$ and $\mathrm{IrO}_{2}$ are considered as the most efficient catalysts for the oxygen evolution reaction (OER) at the anode. However, their large-scale applications are limited by high cost and poor natural abundance. Over the past decades, non-noble metal cobalt-based electrodes have been extensively investigated as promising catalysts for water splitting. For example, Nocera and co-workers have intensively studied $\mathrm{CoP}$ electrocatalysts for OER. ${ }^{2}$ Cobalt-based oxides (such as $\mathrm{Co}_{3} \mathrm{O}_{4}$ nanoparticles and $\mathrm{CoO}_{x}$ amorphous films) were also synthesized and their high catalytic activity toward OER was demonstrated. The formation of binary metals or oxides and the modification of both the physical and chemical properties of the materials are typical strategies used to further improve the electrocatalytic activities..$^{3-5}$ There are reports that introducing a certain amount of Mo can greatly enhance the HER activity of a Co electrode, and a Co-Mo alloy is considered as a promising non-Pt electrocatalyst for HER. ${ }^{6}$ Recently, Co-Mo bimetallic oxides have attracted attention as potential candidates for OER catalysis. Singh et al. synthesized $\alpha$ $\mathrm{CoMoO}_{4}$ oxide by a co-precipitation method and measured its

${ }^{a}$ State Key Laboratory of Oil and Gas Reservoir Geology and Exploitation, Southwest Petroleum University, Chengdu 610500, P. R. China. E-mail: xbge@swpu.edu.cn; Tel: $+86-28-83037337$

${ }^{b}$ The Center of New Energy Materials and Technology, School of Chemistry and Chemical Engineering, Southwest Petroleum University, Chengdu 610500, China

$\dagger$ Electronic supplementary information (ESI) available. See DOI: $10.1039 / \mathrm{c} 7 \mathrm{ra} 00007 \mathrm{c}$ electrocatalytic performance for OER in alkaline medium. ${ }^{7}$ Other Co-Mo based materials with various structures, including amorphous oxide films, porous flowers and 3D ordered microporous architectures, were also fabricated as efficient OER catalysts in the past few years..$^{8-10}$ However, most of these synthetic routes demand conductive supports (e.g. fluorine tin oxide (FTO) or a glassy carbon electrode (GCE), etc.) for catalyst loading, which lead to an increase in fabrication complexity and cost. Herein, we intentionally designed a free standing porous $\mathrm{Co} / \mathrm{Mo}$ architecture prepared by a partial etching process. This etched $\mathrm{Co} / \mathrm{Mo}$ electrode works as an excellent electrocatalyst for both HER and OER in basic and acidic electrolytes. Furthermore, the etching technique reported here for synthesizing porous binary or multi-metal compounds could be extended to other fields like rechargeable metal-air batteries.

\section{Fabrication of $\mathrm{CO}_{3} \mathrm{Mo}$ ribbon, a porous Co/Mo architecture}

The binary $\mathrm{Co}_{3} \mathrm{Mo}$ precursor was made by arc melting using pure Mo and Co. The alloy was re-melted in a quartz tube and subsequently injected onto a rotating $\mathrm{Cu}$ wheel under an argon atmosphere to make $\mathrm{Co}_{3} \mathrm{Mo}$ ribbons with a thickness of $\sim 30 \mu \mathrm{m}$. The porous $\mathrm{Co} / \mathrm{Mo}$ architecture was obtained by immersing the asfabricated $\mathrm{Co}_{3}$ Mo ribbon $\left(0.3 \times 1.2 \mathrm{~cm}^{2}\right)$ into a freshly prepared ceric ammonium nitrate $(\mathrm{CAN})$ aqueous solution $(0.24 \mathrm{M})$ in ambient conditions. After etching for $5 \mathrm{~min}$, the sample was carefully rinsed in DI water and naturally dried in air. A polished commercial cobalt sheet ( $0.2 \mathrm{~mm}, 99.999 \%)$ was also treated using the identical procedures; this sample was denoted as etched Co.

\section{Characterization}

A ZEISS EV0 MA15 scanning electron microscope (SEM) was used to analyse the surface morphologies of the samples. The 
surface electronic states and compositions of materials were studied by analysing the data taken from an X-ray photoelectron spectrometer (XPS) (ESCALAB 250, Thermo Fisher Scientific USA).

\section{Electrochemical measurements}

All electrochemical measurements were performed on an electrochemical workstation (CH Instrument 760E, Shanghai, China) in a standard three-electrode cell. $\mathrm{An} \mathrm{Ag} / \mathrm{AgCl}$ (sat. $\mathrm{KCl}$ ) electrode and a Pt plate were selected as the reference electrode and counter electrode, respectively. The free-standing etched Co and etched $\mathrm{Co} / \mathrm{Mo}$ were applied directly as stationary working electrodes. The OER and HER activities of the electrodes were determined using linear sweep voltammetry (LSV) with a scan rate of $20 \mathrm{mV} \mathrm{s}^{-1}$. For comparison, $\mathrm{Co}_{3} \mathrm{Mo}$ alloy ribbon, and polished Co and Mo sheets were also tested under identical conditions. The current densities were normalized to geometric area and the potentials reported in the text were converted to the reversible hydrogen electrode (RHE) potential according to the equation $E(\mathrm{RHE})=E(\mathrm{Ag} / \mathrm{AgCl})+0.059 \mathrm{pH}+0.197 \mathrm{~V}$. The OER overpotentials $(\eta)$ were calculated using the equation $\eta=$ $E(\mathrm{RHE})-1.23 \mathrm{~V}$.

\section{Results and discussion}

Chemical etching of alloys often results in the formation of porous structures when using a suitable corrosive solution. ${ }^{11,12}$ Here, CAN was selected to partially etch a $\mathrm{Co}_{3} \mathrm{Mo}$ alloy; it has previously been used as a moderate oxidizer to partially oxidize inactive $\mathrm{MnO}$ nanoparticles to active $\mathrm{MnO}_{x}$ catalysts. ${ }^{13}$ For Co sheet, no obvious pores were observed on the surface after CAN etching, as shown in Fig. 1a. The observed particles are Co oxides formed in the oxidizing solution. However, the $\mathrm{Co}_{3} \mathrm{Mo}$ surface was etched into a porous structure, with pore diameters of $c a .300 \mathrm{~nm}$ (Fig. 1b). It is expected that Co atoms on the surface were largely oxidized and dissolved by CAN, thus the remaining Mo atoms self-organized into a porous structure since Mo is highly resistant to corrosion. By employing the optimum etching time of $5 \mathrm{~min}$, the surface composition could be controlled with a Co/Mo ratio of $c a$. 1.4 (from XPS results, Fig. 2).

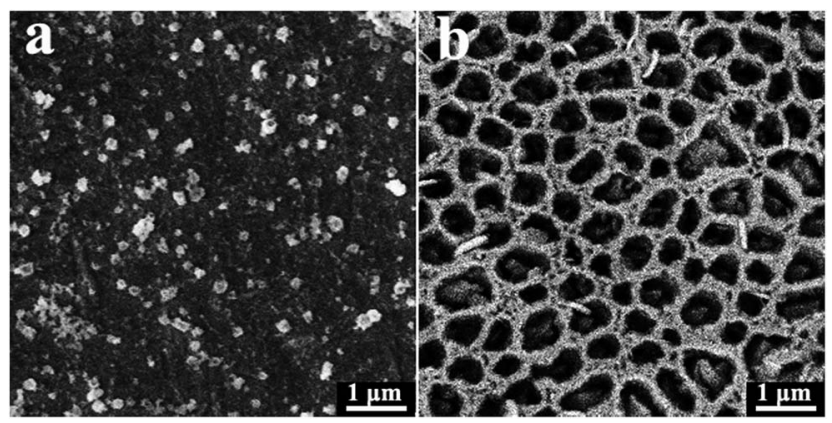

Fig. 1 SEM images of (a) etched Co and (b) etched Co/Mo.
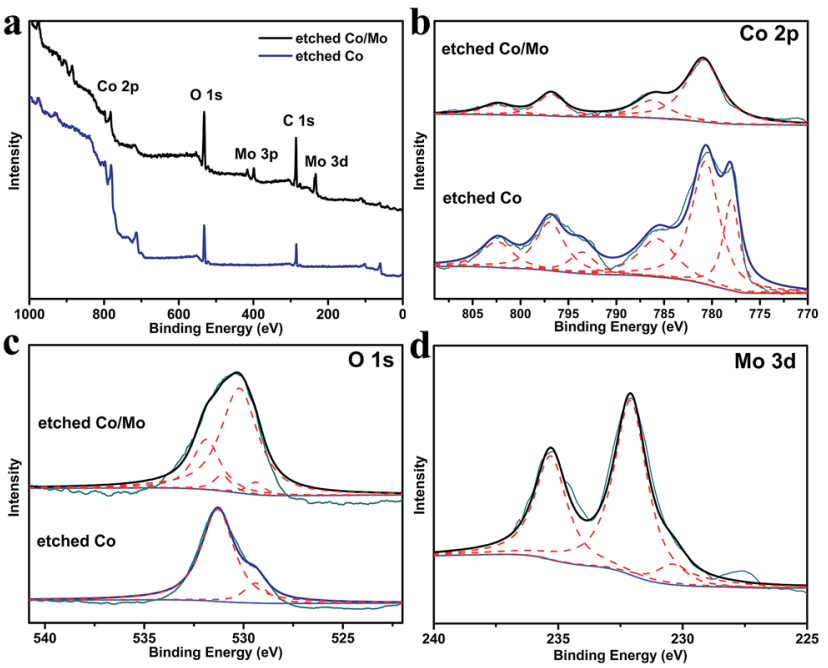

Fig. 2 (a) Survey XPS spectra of etched $\mathrm{Co}$ and etched Co/Mo; high resolution XPS spectra of (b) Co $2 p$, (c) $O$ 1s and (d) Mo $3 d$ in the corresponding catalysts.

The chemical properties of the etched Co and Co/Mo were investigated by XPS (Fig. 2). The XPS survey spectra show the expected photoelectron peaks due to $\mathrm{Co}, \mathrm{Mo}$, and $\mathrm{O}$ elements in the etched Co/Mo sample (Fig. 2a). Fig. 2b shows the high resolution spectra of the Co $2 \mathrm{p}$ region. The observed peaks at $780.8 \mathrm{eV}\left(\mathrm{Co} 2 \mathrm{p}_{3 / 2}\right)$ and $796.6 \mathrm{eV}$ (Co 2p $\mathrm{p}_{1 / 2}$ ) with their neighbouring shakeup satellite peaks could be identified as $\mathrm{Co}^{\mathrm{II}}$ species,${ }^{14}$ indicating that most of the surface Co atoms in both catalysts were oxidized to $\mathrm{Co}^{2+}$ after etching. Meanwhile, metallic Co was also detected on the surface or near-surface of the etched Co sample, with a pair of peaks located at $778.0 \mathrm{eV}$ and $793.5 \mathrm{eV} .^{6}$ No obvious peaks of metallic Co were observed on the etched $\mathrm{Co} / \mathrm{Mo}$ sample, which may result from the aggregation of Mo atoms on the surface during the etching process. The $\mathrm{O} 1 \mathrm{~s}$ spectra (Fig. 2c) for both samples present two peaks at about $529.4 \mathrm{eV}$ and $531.2 \mathrm{eV}$, which could be ascribed to Co-O bonds and hydroxyl groups, respectively. Furthermore, two new peaks at $530.2 \mathrm{eV}$ and $531.9 \mathrm{eV}$ are detected in the etched $\mathrm{Co} / \mathrm{Mo}$. The former corroborates the existence of $\mathrm{Mo}^{{ }^{\mathrm{VI}}-}$ $\mathrm{O},{ }^{10}$ and the latter indicates the presence of a great number of defect sites with low oxygen coordination on the surface of the etched $\mathrm{Co} / \mathrm{Mo}$; such sites have been reported to be favourable for high catalytic activity. ${ }^{15-18}$ The Mo $3 \mathrm{~d}$ spectrum (Fig. 2d) shows a characteristic doublet of $\mathrm{Mo}^{\mathrm{VI}}$ at $232.1 \mathrm{eV}\left(\mathrm{Mo} \mathrm{3d}_{5 / 2}\right)$

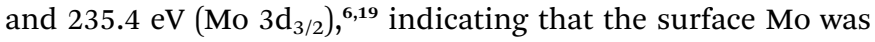
also oxidized by CAN.

Water electrolysis was performed firstly in $0.1 \mathrm{M} \mathrm{KOH}$ to evaluate the OER catalytic activities of the samples, as shown in Fig. 3a. The etched Co/Mo displays a remarkably enhanced catalytic activity toward OER as compared to the pure Co and $\mathrm{Co}_{3} \mathrm{Mo}$ alloy, with an onset overpotential as low as $270 \mathrm{mV}$. The current density of the etched Co/Mo recorded at $1.65 \mathrm{~V} v s$. RHE $(\eta=420 \mathrm{mV})$ is $5.25 \mathrm{~mA} \mathrm{~cm}{ }^{-2}$, more than three times the current density of the $\mathrm{Co}_{3} \mathrm{Mo}$ alloy $\left(1.6 \mathrm{~mA} \mathrm{~cm}^{-2}\right)$ and nearly five times that of the pure Co sample $\left(0.9 \mathrm{~mA} \mathrm{~cm}{ }^{-2}\right)$. The enhanced 

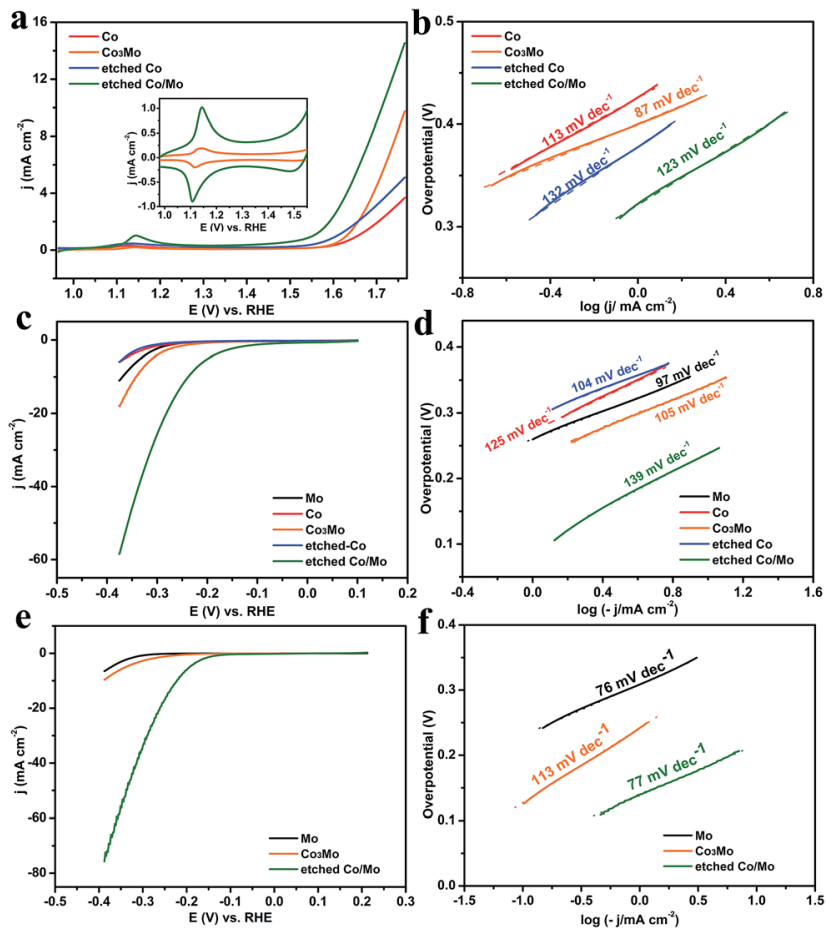

Fig. 3 (a) LSV curves for OER in $0.1 \mathrm{M} \mathrm{KOH}$, and (b) the corresponding Tafel plots; inset in (a) is the magnification of CVs over the potential range of $0.96-1.56 \mathrm{~V}$; (c) LSV curves for HER in $1 \mathrm{M} \mathrm{KOH}$, and (d) the corresponding Tafel plots; (e) LSV curves of $\mathrm{Mo}$ sheet, $\mathrm{CO}_{3} \mathrm{Mo}$ alloy, and etched $\mathrm{Co} / \mathrm{Mo}$ for HER in $0.5 \mathrm{M} \mathrm{H}_{2} \mathrm{SO}_{4}$, and (f) the corresponding Tafel plots.

activity of the etched Co/Mo could be on account of the increased number of active sites and the oxygen vacancies provided by the porous structure after etching, as proved by the SEM and XPS results. This explanation was also confirmed by electrochemical cyclic voltammograms (CVs) as shown in Fig. 3a (inset). The enhanced reversible redox peaks around $1.1 \mathrm{~V}$, ascribed to the conversion of $\mathrm{Co}^{\mathrm{II}} / \mathrm{Co}^{\mathrm{III}}$, suggest that more active Co sites were accessible in the catalysis process. The Tafel slopes were also calculated for evaluating the catalytic kinetics, as shown in Fig. 3b. All samples exhibit slope values of 87$132 \mathrm{mV} \mathrm{dec}^{-1}$, indicating that the rate-determining step is the $\mathrm{M}-\mathrm{OH}$ bond formation $\left(\mathrm{M}+\mathrm{OH}^{-} \leftrightarrow \mathrm{M}-\mathrm{OH}+\mathrm{e}^{-}\right.$, where " $\mathrm{M}$ " denotes the active site). ${ }^{20}$

The $\mathrm{Co}_{3} \mathrm{Mo}$ alloy also works as an efficient catalyst for HER in basic medium, exhibiting a higher activity than those of pure Co and Mo sheet, as shown in Fig. 3c. CAN etching tremendously improves the catalytic activity toward HER. The onset overpotential on the etched $\mathrm{Co} / \mathrm{Mo}$ is about $90 \mathrm{mV}$, overwhelmingly lower than that of the $\mathrm{Co}_{3} \mathrm{Mo}$ alloy $(240 \mathrm{mV})$. The cathodic current density on the etched $\mathrm{Co} / \mathrm{Mo}$ reaches $10 \mathrm{~mA} \mathrm{~cm}{ }^{-2}$ at an overpotential of $\sim 240 \mathrm{mV}$, but the $\mathrm{Co}_{3} \mathrm{Mo}$ alloy requires an overpotential of $\sim 340 \mathrm{mV}$ to give the same current. The Tafel slopes of the Mo sheet, Co sheet, $\mathrm{Co}_{3} \mathrm{Mo}$, etched Co and etched $\mathrm{Co} /$ Mo were calculated to be 97, 125, 105, 104 and $139 \mathrm{mV} \mathrm{dec}^{-1}$ respectively, as presented in Fig. 3d. Additionally, etched Co/Mo showed a superior HER activity in $0.5 \mathrm{M} \mathrm{H}_{2} \mathrm{SO}_{4}$, with an onset

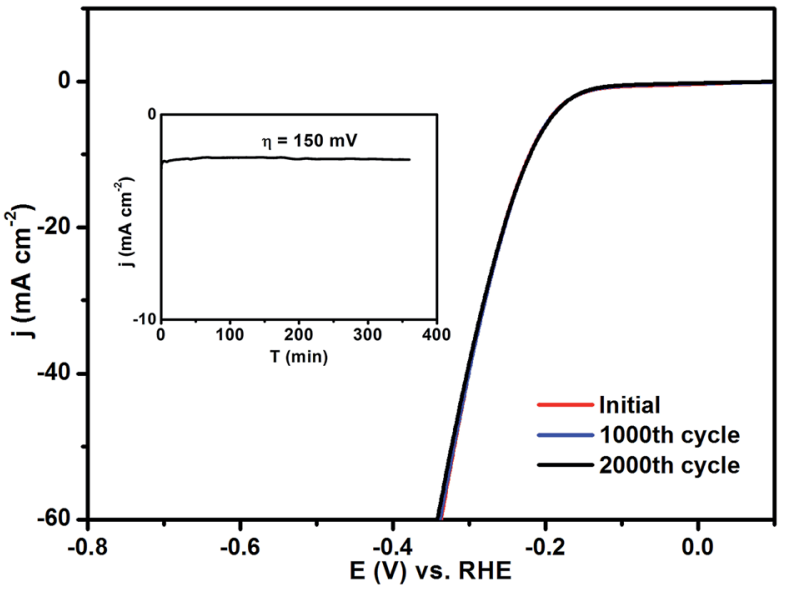

Fig. 4 HER polarization curves of the etched $\mathrm{Co} / \mathrm{Mo}$ before and after 1000 and 2000 cycles in $0.5 \mathrm{M} \mathrm{H}_{2} \mathrm{SO}_{4}$; inset is the chronopotentiometry curve of the etched $\mathrm{Co} / \mathrm{Mo}$ at an overpotential of $150 \mathrm{mV}$.

potential of $109 \mathrm{mV}$ and a Tafel slope of $77 \mathrm{mV} \mathrm{dec}{ }^{-1}$ (Fig. 3e and f). This easily fabricated etched Co/Mo overmatches many of the well-discussed non-noble metal HER electrocatalysts reported to date (see Tables S1-S3, ESI†).

Long-term stability is another important criterion used to evaluate electrocatalysts. Fig. 4 shows the HER polarization curves of the etched Co/Mo catalyst measured before and after 1000 and $2000 \mathrm{CV}$ cycles in $0.5 \mathrm{M} \mathrm{H}_{2} \mathrm{SO}_{4}$. The etched $\mathrm{Co} / \mathrm{Mo}$ catalyst exhibits high stability with no noticeable decay of activity observed. Furthermore, chronopotentiometry measurements were carried out at an overpotential of $150 \mathrm{mV}$ for $6 \mathrm{~h}$, and displayed negligible cathodic current loss (Fig. 4, inset). In alkaline solution, the $\mathrm{Co} / \mathrm{Mo}$ catalyst loses a little current efficiency during extended testing, but a relatively moderate durability toward HER and OER could be observed, as shown in Fig. S3 and S4. $\dagger$ The outstanding catalytic performances for both OER and HER illustrate that the fabricated Co/Mo composite is an excellent bifunctional catalyst toward electrochemical water splitting in various conditions.

\section{Conclusions}

In summary, we describe a CAN etching method to fabricate a free standing porous $\mathrm{Co} / \mathrm{Mo}$ architecture as a highly effective electrode material for water splitting. It is quite stable in longterm electrolysis, especially in acid medium. The unique structure possesses large number of active sites, substantial oxygen vacancies and advantageous charge transport capability, making it a robust electrocatalyst toward OER and HER. This CAN etching technique for synthesizing porous binary or multimetal compounds holds great promise in numerous fields such as rechargeable metal-air batteries and super-capacitors.

\section{Acknowledgements}

This work was supported by the National Natural Science Foundation of China (21403174). 


\section{Notes and references}

1 A. J. Bard and M. A. Fox, Acc. Chem. Res., 1995, 28, 141-145. 2 Y. Surendranath, M. W. Kanan and D. G. Nocera, J. Am. Chem. Soc., 2010, 132, 16501-16509.

3 Y. Xiao, L. Feng, C. Hu, V. Fateev, C. Liu and W. Xing, RSC Adv., 2015, 5, 61900-61905.

4 Y. Tan, H. Wang, P. Liu, Y. Shen, C. Cheng, A. Hirata, T. Fujita, Z. Tang and M. Chen, Energy Environ. Sci., 2016, 9, 2257-2261.

5 T. Grewe, X. Deng, C. Weidenthaler, F. Schüth and H. Tüysüz, Chem. Mater., 2013, 25, 4926-4935.

6 B. Cao, G. M. Veith, J. C. Neuefeind, R. R. Adzic and P. G. Khalifah, J. Am. Chem. Soc., 2013, 135, 19186-19192.

7 R. N. Singh, Madhu, R. Awasthi and A. S. K. Sinha, Electrochim. Acta, 2009, 54, 3020-3025.

8 B. Zhang, X. Wu, F. Li, F. Yu, Y. Wang and L. Sun, Chem.Asian J., 2015, 10, 2228-2233.

9 M. Yu, L. Jiang and H. Yang, Chem. Commun., 2015, 51, 14361-14364.

10 B. B. Li, Y. Q. Liang, X. J. Yang, Z. D. Cui, S. Z. Qiao, S. L. Zhu, Z. Y. Li and K. Yin, Nanoscale, 2015, 7, 16704-16714.
11 Q. Hao, Y. Yu, D. Zhao and C. Xu, J. Mater. Chem. A, 2015, 3, 15944-15950.

12 Z. Zhang, Y. Wang, Z. Qi, W. Zhang, J. Qin and J. Frenzel, J. Phys. Chem. C, 2009, 113, 12629-12636.

13 A. Indra, P. W. Menezes, I. Zaharieva, E. Baktash, J. Pfrommer, M. Schwarze, H. Dau and M. Driess, Angew. Chem., Int. Ed., 2013, 52, 13206-13210.

14 X. Liu, J. Jiang and L. Ai, J. Mater. Chem. A, 2015, 3, 97079713.

15 F. Liang, Y. Yu, W. Zhou, X. Xu and Z. zhu, J. Mater. Chem. A, 2015, 3, 634-640.

16 J. Fan, Z. Chen, H. Shi and G. Zhao, Chem. Commun., 2016, 52, 4290-4293.

17 L. Xu, Q. Jiang, Z. Xiao, X. Li, J. Huo, S. Wang and L. Dai, Angew. Chem., Int. Ed., 2016, 55, 5277-5281.

18 H. Rahaman, K. Barman, S. Jasimuddin and S. K. Ghosh, RSC Adv., 2014, 4, 41976-41981.

19 Z. Li, L. Gao and S. Zheng, Appl. Catal., A, 2002, 236, 163-171.

20 A. T. Marshall and L. Vaisson-Béthune, Electrochem. Commun., 2015, 61, 23-26. 